The book covers cleavage of disulphide linkages ; specific and non-specific cleavage of proteins by enzymes, electrophilic reagents, acid and alkali; fractionation of mixtures of peptides by precipitation, gel filtration, ion exchange chromatography, paper chromatography and electrophoresis; end group determination, ordering the peptide fragments, radioactive techniques; and finally, mass spectrometric and $\mathrm{X}$-ray crystallographic techniques. The latter techniques have for good reasons been discussed only briefly, but sufficient information is provided to make possible a useful comparison of data obtained by X-ray analysis with those obtained by conventional chemical techniques. At the beginning of each chapter the principles involved are stated concisely in a homely style and these are followed by practical details of experimental procedures. An excellent job has been done in selecting these practical examples, most of them allowing the worker to take his problem directly to the bench without reference to the original literature. A list of sources is, however, available at the end of each section.

The book is not overburdened with tables and figures, and abounds in sound advice and hints on how to avoid pitfalls. It forms a companion volume to the author's earlier Amino Acid Determination: Methods and Techniques, but can be read and used independently. The level of proof reading was quite good although a curious chapter heading was missed on page VIII. The publisher could have been a little more generous with the subject index. Overall the book fulfils its aims and should be well received by research workers who have an interest in peptide and protein sequence data.

J. LEgGetT BaIley

\section{Chain Genetics}

The Genetic Markers of Human Immunoglobulins. By R. Grubb. (Molecular Biology, Biochemistry and Biophysics, Vol. 8.) Pp. xii 152. (Chapman and Hall: London; Springer-Verlag: Berlin and New York, November 1970.) $£ 4.90$.

THE immunoglobulins (Igs) are unique among proteins in their diversity of structure and function. The discovery in 1956 of genetic markers on human and rabbit Igs by the author of this book and by Oudin, respectively, was therefore of particular significance. The subsequent intensive study of these markers has provided an essential framework for understanding the genetic control of antibody synthesis, and has delineated several features of great interest with regard to the assembly of antibody molecules and the differentiation and cooperation of cells which produce antibodies. Genetic markers have also been useful in studies of the phylogeny, ethnic features and ontogeny of immunoglobulins, and forensic pathologists have used them with practical effect in cases of disputed pater ty and confusion of newborn infants in maternity hospitals. They can be of unique value in the latter case because the infant's genotype for $\mathbf{I g}$ synthesis is not expressed at birth and its circulating IgG is for practical purposes exclusively of maternal origin.

Genetic markers are now known for three of the seven major classes of immunoglobulin heavy and light chains. Identification of their many polymorphic forms involves the use of human antisera which specifically agglutinate red blood cells coated with human immunoglobulin. The antihuman $\gamma$-globulins used in these tests originate principally in patients who have had multiple blood transfusions or in subjects who may be apparently healthy or afflicted with rheumatoid arthritis. The clinical significance of these antibodies in the latter context and their possible role in the pathogenesis of disease are matters of continuing controversy and interest.

All these intriguing ramifications of an important topic are lucidly outlined in the first eighty-five pages of this admirable, small book. The text is supported by a wealth of tabulated data and a concluding appendix of thirty pages provides a comprehensive source for the original references, mode of detection, ethnic distribution, molecular localization and inheritance of some thirty genetic factors which have been detected on human immunoglobulins. This book will be of great value as a succinct and authoritative source for immunologists, geneticists, clinicians and others interested in the many sided problems of antibody synthesis. It is regrettable that the author's economy and accuracy were not emulated by the publishers who are responsible for the inflated price and several printing errors -some diverting, such as that on page 82 , which concerns "the mouse lamb dalight chains" of IgM. S. COHEN

\section{Vibrations}

Low-Frequency Vibrations of Inorganic and Coordination Compounds. By John R. Ferraro. Pp. xiv +309. (Plenum: New York, 1971.)

IMPORTANT developments in far infrared instrumentation during the past ten years have led to a considerable increase in the published data on low frequency vibrations of molecules and ions, which are not only of interest to spectro- scopists but of potential value to other chemists. In this book the author, who has published a considerable number of research papers in this field, sets out to demonstrate the usefulness to inorganic chemists of infrared absorption measurements below $650 \mathrm{~cm}^{-1}$ on inorganic compounds.

A brief introduction sketches the history of far infrared measurements and the experimental and interpretative problems of this region of the spectrum, and is followed by a chapter describing commercial instruments including interferometers, some in considerable detail but others only in briefest outline. A comparison of the relative merits of spectrometers and interferometers, probably of greater general interest than the rest of the chapter, is unfortunately presented in brief tabular form, with references to other texts. The third chapter summarizes useful data on window materials and some common organic solvents. Calibration is also briefly discussed but one is referred to other texts for many of the calibration data. The fourth chapter sketches the use of inelastic neutron scattering and high pressure techniques in vibrational studies, again with references to other sources for details.

The next four chapters, comprising more than two-thirds of the book, present data (including those from Raman measurements) and discussion on metaloxygen, metal-halogen, metal-nitrogen and miscellaneous metal-ligand vibrations. Many of the data are in tabular form and the presentation is similar to that in other books of this type. The final chapter very briefly summarizes some results on ion-pair and lattice vibrations, clathrate vibrations and low frequency electronic transitions. Two appendices give tables of crystal site symmetries and subgroup correlations, and describe a method for factor group analysis of crystal vibrations.

This book undertakes to cover a very wide field embracing almost the whole of metal-ligand vibrations, which is scarcely reduced by the rather artificial limitation to frequencies below 650 $\mathrm{cm}^{-1}$. In order to save space much use is made of references to other texts for detailed discussion, and on many topics no detailed or critical account is given. References are included up to 1969 , but, the author says, the book does not claim complete coverage of topics or references, and there are some obvious gaps. The book therefore seems to be chiefly of value to newcomers and non-specialists in this field, giving in one volume brief accounts of both experimental and interpretative matters. While also of use to specialists as a recent source of references, its value as a convenient source of data is somewhat reduced by its lack of comprehensiveness on details. J. A. Creighton 